\title{
Incomplete pairwise comparative judgments: Recent developments and a proposed method
}

\author{
Melfi A. Alrasheedia*
}

${ }^{a}$ Department of Quantitative Methods, School of Business, King Faisal University, Hofuf, Saudi Arabia

\begin{tabular}{l}
\hline C H R O N I C L E \\
\hline Article history: \\
Received December 2, 2018 \\
Received in revised format: \\
January 26, 2019 \\
Accepted January 27, 2019 \\
Available online \\
January 28, 2019 \\
\hline Keywords: \\
Decision analysis \\
Diagonal sampling \\
Incomplete judgments \\
MCDM \\
Pairwise comparisons
\end{tabular}

C 2018 by the authors; licensee Growing Science, Canada.

\section{Introduction}

Most of the multi-criteria decision-making approaches consist of ranking or weighing a set of alternatives with regard to a set of criteria (Lootsma, 1999; Toloie-Eshlaghy \& Homayonfar, 2011). Among the existing methods, the pairwise comparison ('PW') approach is commonly used and is attractive as the desired decision is broken down into smaller sub-decisions on two alternatives at a time, there is a possibility of checking the internal logic of the comparisons and thus commenting on the quality of the overall decision, and there are well-known mathematical models for the additive model which enable the comparisons to be synthesized into the desired decision (Foster, 1994). This model suffers none of the limitations and deficiencies that other Analytical hierarchy Process (AHP) (Saaty, 1980) and multiplicative models face (Foster, 2001, Alrasheedy, 2003). The PW technique is used to produce sets of PW judgment matrices where the elements of each matrix represent the decision maker's estimate on the preference of one alternative over another with respect to a given criterion. A PW matrix is filled by the decision maker by answering questions such as: "On a scale from -8 to 8 , how much do you prefer the alternative option $i$ compared to the alternative option $j$ ?" The answer to the question is noted as $a_{i j}$ and gives a weight difference $w_{i}-w_{j} \approx a_{i j}$. Then, these answers are saved into a PW matrix $\mathbf{A}$ as follows:

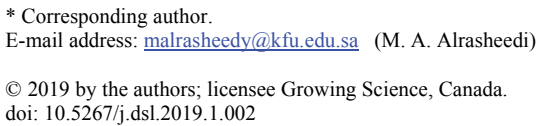




$$
A=\left[\begin{array}{ccccc}
0 & a_{12} & a_{13} & \ldots & a_{1 n} \\
-a_{12} & 0 & a_{23} & \ldots & a_{2 n} \\
-a_{13} & -a_{23} & 0 & \ldots & a_{3 n} \\
\vdots & \vdots & \vdots & \ddots & \vdots \\
-a_{1 n} & -a_{2 n} & -a_{3 n} & \ldots & 0
\end{array}\right] \approx\left[\begin{array}{ccccc}
w_{1}-w_{1} & w_{1}-w_{2} & w_{1}-w_{3} & \ldots & w_{1}-w_{n} \\
w_{2}-w_{1} & w_{2}-w_{2} & w_{2}-w_{3} & \ldots & w_{2}-w_{n} \\
w_{3}-w_{1} & w_{3}-w_{2} & w_{3}-w_{3} & \ldots & w_{3}-w_{n} \\
\vdots & \vdots & \vdots & \ddots & \vdots \\
w_{n}-w_{1} & w_{n}-w_{2} & w_{n}-w_{3} & \ldots & w_{n}-w_{n}
\end{array}\right]
$$

A PW matrix is referred to as consistent if the transitivity $a_{i j}+a_{j k}=a_{i k}$ holds for all indices $i, j=$ $1, \ldots, n$. Otherwise, the matrix is referred to as inconsistent. In real decision problems, some different levels of inconsistency are acceptable (Bozaki et al., 2010). In general, the PW method consists of an ordinal and a cardinal question. The ordinal question asks which alternative $A_{i}$ or $A_{j}$ is more desirable, and the cardinal question asks for the number of units that the desired alternative is preferred to over the other. When applied to a set of $n$ alternatives $\left\{a_{1}, \ldots, a_{n}\right\}$ with respect to a single criterion, the number of comparisons that have to be made is $n(n-1) / 2$. The response of a decision maker to the relative ordinal question is the main input to find the comparison matrix. With the information, it is possible to compute the weights, the performance scores for every alternative option on each criterion, and the overall alternative option required by the decision maker. When the information of the cells of the comparison matrix is complete, comparisons can be performed, and it is a case of decision making with complete information. However, if there are empty cells due to uncertainties or lack of information it is not possible to make certain judgments, and the decision-making is a case of incomplete information with missing comparisons.

The PW technique is used in many multi-criteria decision settings, such as in engineering, education, social and political issues, personal and management selection, natural disasters, environmental topics, energy alternatives, military affairs, suppliers selection, store location, public transportation, health care, sports evaluation, finance provision, questionnaire surveys and other areas (Hotman, 2005; Zhang et al., 2012; Makui et al., 2010; Lolli, et al., 2014; Schmidt et al., 2015; Han, 2016; Ergu et al., 2016; Kahraman et al., 2016; Um, 2016; Song \& Kang, 2016; Inti \& Tandom, 2017; Joneghani \& Joneghani, 2018; Zhou et al., 2018). In particular, an interesting application of the technique is through survey questionnaires where respondents play the role of decision makers with the goal of selecting the best product/service by giving strengths of preferences regarding a list of alternatives. The Internet has become an increasingly attractive way of collecting comparative data, making decisions on consumer behavior or marketing studies with the aim of getting reliable responses describing the respondents' true preferences (Akarte et al., 2001). On the surface, the attractive characteristics of PW technique should lead to increased reliability and confidence in the decision-making process. However, in many practical applications such as survey questionnaires, the number of alternatives or criteria considered in the decision problem is often relatively large. A respondent needing to evaluate 15 alternatives using the method would be faced with having to make 105 comparisons. The subsequent task of making the comparisons would be cumbersome, tiring and time-consuming for the respondents and may cloud judgment. Consequently, the decisions become unreliable and conclusions from them are problematic. Furthermore, it is widely acknowledged that a lengthy survey and questionnaires yield a small response and low usability rate (Alrasheedy, 2003). Due to the problem, in the current work, a method is proposed to reduce the number of alternatives or pairwise comparisons in the decision problem. The method is called the linear model which is a synthesis of the works done by Barzalai (1987), Foster (1994), and Lootsma (1993). The model relies on an additive comparison scale between 0 and 8 (indifference $=0$, weak preference $=+2$ or $-2, \ldots$, absolute preference $=+8$ or -8 ). The model has advantages, theoretical and practical over other models (Foster, 1997). On the theoretical side, the model is mathematically transparent as it is axiomatically based and uses well understood linear models and avoids problems of normalization and aggregations surrounding some popular models such as the Analytic Hierarchy Process ('AHP') of Saaty (Lootsma, 1999; Barzalai et al., 1997; Foster, 1994). The 
practical advantages include simple methods of scoring and aggregation and the use of 2-diagonal sampling plans.

The aim of the current paper is threefold. The first is to review the recent developments in incomplete decision matrices mostly with AHP. The second is to represent simple sampling formulae for finding scores or priorities and consistencies which reduce the total number of comparisons from $n(n-1) / 2$ to $2 n$ while maintaining the same quality of results (Foster, 1997; Foster, 2001). A third goal is to revisit a numerical example proposed in the literature and compare the obtained results to show the effectiveness of the proposed technique in a case of incomplete information matrices (Ergu et al., 2016).

\section{Literature review}

PW technique has been used in a number of research settings and has been found to be a reliable method for obtaining relative judgments in a variety of applications (Lootsma, 1999). The analysis of multicriteria decision making in complex problems tends to cause complications due to an excessive number of PWs to be performed and for the difficulties in finding missing comparisons in incomplete information matrices. The problems have received considerable attention from researchers in this field, and within the perspective of the current review, estimation of missing comparisons is first considered and then are the efforts to reduce the number of PWs. With regard to the estimation of missing comparisons, there are several works published in the literature that investigate this topic (Harker, 1987a,b,c; Carmone et al., 1997; Fedrizzi \& Giove, 2007; Bozoki, 2010; Bozoki et al., 2010; Peng et al., 2011; Zhang et al., 2012; Wang et al., 2012; Xu et al., 2013; Srdjevic et al., 2014; Bochkov \& Zhigirev, 2014; Benitez et al., 2015; Chen et al., 2015; Um, 2016; Ergu et al., 2016; Wang et al., 2016; Tekile \& Bozóki, 2017; Al Salem, 2017; Zhou et al., 2018). Next, some of the most recent articles have examined to obtain an idea of the current developments. Bozoki et al. (2010) investigated the application of the eigenvector method and the logarithmic least squares method in the analysis of incomplete pairwise comparison matrices. In this sense, the researchers studied the uniqueness of the best completion matrix with both methods and found the same graph structure representative of uniqueness. Various numerical examples explained their findings. Similarly, Bozoki (2010) described the pairwise comparison matrix and the positive weight vector and discussed three methods: the eigenvector method, the least squares method, and the logarithmic least squares method. The scholar also associated graph structures to complete and incomplete pairwise comparison matrices and suggested a conjecture to the optimal solution of the incomplete pairwise matrix when using the least square method.

Peng et al. (2011) investigated a distinct topic related to the questionnaire survey. The researchers employed a scale format to organize the surveyed items for the comparison matrix and proposed an Induced Bias Matrix Model ('IBMM') to evaluate the missing values scores in an incomplete matrix. The purpose of the optimization process was to reduce the number of the scored items in the questionnaire survey, and they provided an example to illustrate their results. Analogously, Srdjevic et al. (2014) evaluated missing comparisons by the First Level Transitivity Rule ('FLTR') method. The authors compared the results with estimations obtained by Harker's and van Uden's methods. The results were similar; however, according to the authors, the FLTR guarantees coherency owing to the missing elements had derived from the entries of the same matrix. In another context, Bochkov and Zhigirev (2014) studied the decision making when the information about the objects was unavailable or uncertain, and it was not possible to make PWs of the objects under study. To solve the problem, they gave equal initial weights to the compared objects, and through a minimization process involving graph theory the weights approximate to the true value within a specified tolerance. Um (2016) also studied the uncertainty and the stochastic nature of some decision parameters in supplier evaluation and selection using AHP combined with uncertainty theory based on a fuzzy technique. A computer program allowed finding the best supplier among a number of options according to several criteria. A numerical example validated the efficacy of this method. Considering another decision-making process 
relating to emergency management in the case of natural disasters, Ergu et al. (2016) analyzed the reduction of pairwise comparisons by using the Geometric Mean Induced Bias Matrix ('GMIBM') technique combined with the least square method and the least absolute error. The researchers solved a numerical example, and a real case related to the Yushu earthquake. In a different ambit, Tekile and Bozóki (2017) applied the eigenvector method and the logarithmic least squares method to analyze the incomplete pairwise comparison matrix to rank top table tennis players by using data from the International Tennis Federation. Studies also indicated that consistency properties were essential to estimate weights and missing values of incomplete matrices (Wang et al., 2012 \& Wang \& Li, 2015). When analyzing the possibility of reducing the total number of PWs, it can be demonstrated that when applied to a set of $n$ alternatives, the total number of comparisons that have to be made using the classical Saaty's procedure is $n(n-1) / 2$ (Saaty 1980). However, as mentioned, one drawback of the technique is that given a large number of alternatives (e.g., alternative $\geq 6$ ) then a large number of comparisons are required. Such can lead to information overload for the decision maker resulting in fatigue and boredom or simply loss of interest in the decision process itself (Carmone et al., 1997 \& Foster, 1996). Lengthy surveys and questionnaires yield a small response and low usability rate since it requires a significant time commitment from the hapless respondents (Alrasheedy, 2003). To find a method for reducing the total number of comparisons while preserving the same quality of results is of great practical importance. In the literature, some approaches that analyze the issues arise from a large number of comparisons (Harker, 1987a, 1987b, 1987c; Millet \& Harker, 1990; Wedley, 1993; Wedley et al., 1993; Triantaphyllou, 1999; Ra, 1999; Hotman, 2005; Ramik, 2013; Jalao et.al., 2014; Koczkodaj \& Szybowski, 2015; Ergu et al., 2016; Song \& Kang, 2016; Han, 2016; Inti \& Tandom, 2017). Generally, the attempts of previous researchers (Harker 1987a, 1987b; Millet \& Harker, 1990; Wedley, 1993; Wedley et al., 1993) were based on methods such as AHP and the least squares method that lead to sampling techniques which were elaborated and technical. Other methodologies were presented in an excellent investigation carried out by Carmone et al. (1997) who reviewed the existing methods and conducted simulations to compare the performance. Also, trying to solve the problem, Triantaphyllou (1999) introduced a dual formulation in which instead of comparing two alternatives at a time under a given criterion as in the traditional way, the decision maker compared how well two performed within a single alternative at a time without skipping any comparison. The technique was dependent on the number of criteria and changes the way of eliciting pairwise comparisons. The dependency limits its practicality. Using a different approach, $\mathrm{Ra}$ (1999) developed a heuristic shortcut technique using the geometric mean approach which he called chain-wise paired comparisons. However, a single error may have a significant impact and lead to the wrong ranking of the alternative, hence jeopardizing the benefits of having redundant ones. Subsequently, Hotman (2005) proposed to reduce the PWs in an engineering process selection of the best microencapsulation technique of small amount of substances by using the so-called Base Reference AHP (BR-AHP). For the case of 5 criteria and 6 alternatives, 35 pairwise comparisons are required by using BR-AHP, but, if we use standard AHP, we need 85 comparisons. In a different way, Jalao et al. (2014) used a Binary Integer Program (BIP) to decompose the pairwise comparison matrix into various subsets employing interdependence scores. As a result of the decomposition, the number and the inconsistency of the pairwise comparisons were reduced. The researchers presented an example to show the capability of the method. Alternatively, Koczkodaj and Szybowski (2015) suggested that using the notion of generators the number of pairwise comparisons can be reduced from $n(n-1)$ to (n-1). Matrix and graph theories were used to reconstruct a consistent $n \times n$ pairwise comparison matrix from (n-1) entries or generators placed in specific locations. Some numerical examples were exposed to clarify their findings. In a setting related to military purposes, Song and Kang, (2016) indicated that the number of comparisons is reduced by assigning weights according to a hierarchy structure and consistency ratio. The method was applied using a survey for the valuation of a weapon system research and development project. Alternatively, in a sports match decision-making problem, Han (2016) indicated that the amount of PWs grows as the number of alternatives increases and suggested that the number of pairwise comparisons could be reduced if comparisons were shared with multiple decision makers under a consensus process. Also, Ergu et al. (2016) analyzed the reduction of PWs to respond to emergencies 
as fast as possible and to estimate the missing comparisons. In the field of engineering, Inti and Tandom (2017) reduced inconsistency and the number of inputs or pairwise comparisons employing additive transitive fuzzy preference relations in their analysis. They applied the proposed method to evaluate the sustainability of transportation infrastructure. It is worth commenting that Kou et al. (2015) made an extensive review of various relevant aspects in the analysis of pairwise comparison matrices such as measurement scales, consistent index, cardinal, and ordinal inconsistency, missing data estimation, priority derivation methods and made some remarks about the reduction of pairwise comparisons.

With the examination of the current literature, an overall view of some of the tendencies is used to reduce the number of PWs and to find missing comparisons in incomplete information matrices which are the main topics considered in the present paper. In the context, the current article proposes a sampling methodology based on a linear space model that can reduce the number of comparisons from $n(n-1) / 2$ to $2 n$. The sampling is based on optimizing the consistency of skew-symmetric matrices on the linear space. The paper is structured as follows. First, diagonal samples and sample spaces are introduced. Next, a linear equation is derived for the completed sample matrix and the scores of the alternatives which allow us to apply diagonal samples and to work with circulant matrices. Subsequently, a more explicit expression which allows modeling and calculations is presented with examples. Then, the consistency of sampled matrices and the results of computer simulations are discussed. Finally, an example from the literature (Ergu et al., 2016) is solved, and the results are compared to demonstrate the capability of the method proposed.

\section{Results and discussion}

\subsection{Diagonal Samples}

It is theoretically sufficient to take only a subset of all possible comparisons to complete the whole set of comparisons. Particular terms such as "missing" or "incomplete" PWs have been used mostly in the literature, but to the best of our knowledge, the term "sampling" has been used by Alrasheedy (2003). However, the terms mean the same thing. That is, they try to deduce from a sample or incomplete/missing comparisons matrix the implied weights or scores of the alternative options. Throughout this paper, we use the term "sampling". Our sampling is not random; it is controlled by diagonal sampling. The sample is called the $k$-diagonal samples because the corresponding comparisons matrix has $k$ (usually here $k=2$ ) diagonals used as the sample (apart from the main diagonal). One advantage is that it allows consistency to be checked. Furthermore, the sampling plan has two important characteristics: 1) being unbiased; since each alternative is involved in the same number of comparisons as the other, and 2) being balanced; since each alternative occurs as the first element in the comparison the same number of times as it occurs as the second. The characteristics are guaranteed in the proposed 2-diagonal sampling approach while they may not be satisfied with other sampling techniques and attempts mentioned earlier. A $k$-diagonal sample is given by:

1. Randomly numbering the options,

2. Fixing $k \in n, 2 \leq k \leq\left[\frac{n-1}{2}\right]$

where $n=$ number of options, $k=$ number of diagonals,

3. Comparing $i$ with $((i+j-1) \bmod n)+1, i=1, \ldots, n ; j=1, \ldots, k$.

The number of comparisons to be made under this sampling scheme is $k \times n$. In applications, $k$ is usually 2 . Thus, for 15 alternatives the sample requires 30 comparisons to be made as against 105 for the case of complete judgment matrices. For instance, if $k=2$, the sampling will take the form as shown below that present sample comparison matrices for $n=8$ with $k=2$ and 3 . 


$$
\left[\begin{array}{cccccccc}
0 & \mathrm{~K}=2 \\
0 & -3 & -7 & 0 & 0 & 0 & 2 & 5 \\
3 & 0 & -8 & -7 & 0 & 0 & 0 & -6 \\
7 & 8 & 0 & -5 & -5 & 0 & 0 & 0 \\
0 & 7 & 5 & 0 & -2 & -5 & 0 & 0 \\
0 & 0 & 5 & 2 & 0 & -6 & -3 & 0 \\
0 & 0 & 0 & 5 & 6 & 0 & 7 & 3 \\
-2 & 0 & 0 & 0 & 3 & -7 & 0 & 4 \\
-5 & 6 & 0 & 0 & 0 & -3 & -4 & 0
\end{array}\right] \quad\left[\begin{array}{cccccccc}
0 & -3 & -7 & 3 & 0 & -4 & 2 & 5 \\
3 & 0 & -8 & -7 & 0 & 0 & -5 & -6 \\
7 & 8 & 0 & -5 & -5 & -4 & 0 & -7 \\
-3 & 7 & 5 & 0 & -2 & -5 & 7 & 0 \\
0 & -6 & 5 & 2 & 0 & -6 & -3 & 7 \\
4 & 0 & 4 & 5 & 6 & 0 & 7 & 3 \\
-2 & 5 & 0 & -7 & 3 & -7 & 0 & 4 \\
-5 & 6 & 7 & 0 & -7 & -3 & -4 & 0
\end{array}\right]
$$

It can be seen that the upper half of each sample contains $2 \times 8(3 \times 8)$ entries and that the lower half is merely skew-symmetric to the upper. The samples contain two (three) diagonals of information.

\subsection{Sample Spaces}

Following from the theory of the linear model, Foster (1996) introduced three sample spaces.

1. Given a space or subset $\Psi \in I(n) \times \mathrm{I}(n)$ for all possible comparisons, $\{i, j\} \in \Psi$ if alternative $i$ is compared with the alternative $j$ using a symmetric, but not reflective relation. That is $\{i, j\} \in \Psi \Rightarrow\{j, i\} \in \Psi$ but $\{\mathrm{i}, \mathrm{j}\} \notin \Psi, \forall \mathrm{i}$.

2. Given a sample space $\Psi$, we also have the complementary non-reflexive symmetric sample space $\Psi^{c}=\{\{i, j\} \notin \Psi\}-\{\{i, i\}\}$.

3. Given a sample space $\Psi$, we define the following subspace of $\Sigma_{n}$ :

$$
\mathrm{S}(\Psi)=\left\{\mathrm{A}=a_{i j} \in \Sigma_{n}:\{i, j\} \notin \Psi \Rightarrow a_{i j}=0\right\}
$$

i.e. all comparisons which are not in the sample are given the value of 0 .

Note that these two space $\Psi, \Psi^{c}$ are orthogonal complements of each other under the Frobenius norm.

\subsection{Completing a sample of comparisons}

We see how to complete a sample space $\Psi$ of comparisons by adopting the following axiom:

Axiom 1 (Foster, 1997)

Giving a matrix $\mathrm{A}=a_{i j} \in \mathrm{S}(\Psi)$ we are going to find the nearest matrix $\mathrm{C}=c_{i j} \in C_{n}$ to $A$ which has the same comparisons as $\Psi i,\{i, j\} \in \Psi \Rightarrow c_{i j}=a_{i j}$. Then the scores of $A$ are given by the scores of $C$. Comparisons in $A$ are kept without change. Axiom 1 is equivalent to completing the matrix by finding the nearest consistent matrix with the original entries. This problem of completing a given sample matrix is transformed to finding the matrix $B=b_{i j} \in \mathrm{S}\left(\Psi^{c}\right)$ such that $C=A+B$ and $\mathrm{d}\left(C, C_{n}\right)$ is a minimum where $\mathrm{d}($, $)$ is the Frobenius matrix. From now on, we assume that $\mathrm{A}=\left(a_{i j}\right) \in \Sigma_{n}$ is fixed (Foster, 1997). Working on sample space, the nearest consistent matrix to $A$ in $C_{n}$ is given by, $A_{I}=\left(w_{i}-w_{j}\right), w_{i}=\frac{1}{n} \sum_{p,\{i, p\} \in \Psi} a_{i p}$. Now let, $\mathrm{B}=b_{i j}, \mathrm{D}=\left(y_{i}-y_{j}\right)$.

Lemma 1 (Foster, 1997)

$\mathrm{d}\left(\mathrm{A}+\mathrm{B}, C_{n}\right)$ is a minimum for $\mathrm{B} \in \mathrm{S}\left(\Psi^{c}\right)$ iff $\exists \mathrm{D} \in C_{n}$ such that $\|B-D\|^{2}-2<A_{1}, D>$ is a minimum.

Let $q_{i}=\{\{j:\{i, j\} \in \Psi\} \mid$ be the number of other alternative options to which $i$ is compared within the sample $\Psi$. The concern now is how to generate the matrix B. According to the linear model theory, the corresponding weights are derived from a convex quadratic function, and we can easily find an 
optimal solution by solving the associated set of normal equations. In order words, the problem of completing a k-diagonal sample matrix may be reduced to solving a system of linear equations as described by the following theorem.

Theorem 1 (Foster, 1997)

Let $B=b_{i j} \in S\left(\Psi^{c}\right)$ where $b_{i j}=y_{i}-y_{j}, \forall\{i, j\} \notin \Psi$ and such that $\left(y_{1}, \ldots, y_{n}\right)$ satisfy the following system of the linear equation:

$$
q_{i} y_{i}-\sum_{(i, j) \in \Psi} a_{i j} y_{j}=n w_{i}, i=1, \ldots, n
$$

However, k- diagonal samples have linear equations of maximal rank $n-1$. Thus the solution is unique up to an additive constant. According to Foster (1994), the assumption of maximal $n-1$ is equivalent to assuming that the sample $\Psi$ is completable, i.e. given alternative options $i, j$ there is a chain of comparisons from $i$ to $j$.

\subsection{K-Diagonal Samples and Circulant Matrices}

\subsubsection{K-Diagonal Samples}

The k-diagonal samples introduced earlier can be reconsidered in order to illustrate how to complete the sample and consequently obtain the scores of the options.

Fix $k \in I(n), 2 \leq k \leq\left[\frac{n}{2}\right]-1, n \geq 6$. Let $\Psi=\{(i, i+j-1) \bmod n+1\}: i=1, \ldots n ; j=1, \ldots, k$. In this case, we have $q_{i}=2 k, i=1, \ldots, n$.

Let $\Pi=P=\left(p_{i j}\right), p_{i j}=\left\{\begin{array}{cc}1 & j-i=1 \bmod n \\ 0 & \text { otherwise }\end{array}\right.$ be the permutation matrix of order $n$. The system of equations given by the Theorem above can be formulated in a matrix form in the following way:

$Q y^{T}=n w^{T}$ where $y=\left(y_{1}, \ldots, y_{n}\right), w=\left(w_{1}, \ldots, w_{n}\right), Q=\sum_{j=1}^{k}\left(2 I_{n}-\left(\Pi^{j}-\Pi^{-j}\right)\right)$ and $Q$ is a singular symmetric circulant matrix of rank $n-1$ and $I_{n}$ is the identity matrix.

\subsubsection{Circulant Matrices}

We have chosen to work with circulant matrices (or just circulants) because the properties are well known and easily derived (Davis, 1994). They are an especially tractable class of matrices in which inverses, products, and sums are also circulant and hence both straightforward to construct.

A $n \times n$ matrix $A_{n}=\left(a_{j k}\right)^{n-1}$ is called a circulant if $a_{i j}=a_{j-k}(\bmod n)$. The structure can also be characterized by circ $\left[a_{0}, \ldots, a_{n-1}\right]=\sum_{i=0}^{n-1} a_{i} \Pi^{i}$. Thus, a circulant is completely determined by its first row, i.e., each row is a cyclic shift of the row above it.

\subsubsection{Modeling and calculation of scores}

The Theorem above works well from a theoretical point of view. However, it is possible to derive a more explicit expression for the comparisons, not in the sample, if we apply properties of diagonal samples and circular matrices (and particularly the inverses of circulants) to the Theorem above.

In Section 3.4.1 we may rewrite Theorem 1 as $Q y^{T}=n w^{T}$.

Two important issues are: 
1. $\Pi^{n}=I_{n}$ in the equivalent polynomial form $x^{n}=1$.

2. We assume that the weights of $y$ are normalized so that $\sum_{i=1}^{n y} y_{i}=0$.

3. Both are shown to be the case by Foster (1996). The following theorem gives a fast and effective way to find the vector of scores $y$.

\section{Theorem 2 (Foster 1996)}

1. $Q=Q_{1}\left(\Pi+\Pi^{-1}-2 I_{n}\right)$, where $Q_{1}=\sum_{i=2}^{k}\left(\frac{(k+i)(k-i+1)}{2}\left(\Pi^{k-i+1}+\Pi^{n-(k-i+1)}\right)\right)+\frac{k(k+1)}{2} \sum_{i=0}^{n=2 k}\left(\Pi^{k+1}\right)$

2. $y^{T}=D_{n . k} w^{T}, D_{n, k}=-\frac{1}{n} S R Q_{1}^{-1}, S=\sum_{i=1}^{n} i \Pi^{i-1}$ and $R=\sum_{i=2}^{n}(n-i+1) \Pi^{i-1}$

Obviously, the conclusions obtained above also apply here. In addition, the following observations which are important in terms of computations can be made:

1. The matrices $S$ and $R$ are circulants that depend only on $n$, the number of options in the problem at hand.

2. $Q_{1}$ and hence $Q_{1}^{-1}$ are circulant both dependent on $n$ and $k$, the number of diagonal in the sample.

3. From 1 and 2, the matrix $D_{n . k}$ is circulant and is independent of the sample matrix at hand, i.e., is fixed for any decision problem of prescribed $n$ and $k$.

4. Only the first row of the output of $D_{n . k}$ needs to be stored in a computer implementation.

5. The sample matrix row means vector $w$ contains all the information on the sample.

Observing the above facts then computing the matrices $D_{n . k}$ requires the following four procedures to be implemented in our algorithm:

a) A function that converts a list of numbers into the circulant matrix.

b) A function that takes a polynomial of degree $n-1$ in the variable $x$ and produces the list of coefficients.

c) A procedure that directly computes the matrix $Q_{1}$ which depends on $n$ and $k$.

d) A procedure that directly computes an intermediate matrix $T=-S R$ which only depends on $n$ the expression for $D_{n . k}$. This circulant $T$ is defined in matrix form.

\section{Example:}

By using Theorem 2 along with the observations and procedures set out, it is straightforward to verify the following outputs:

$$
D_{8,2}=\left[\begin{array}{cccccccc}
0.8965 & -0.7928 & -0.9124 & -1.3648 & -1.3875 & -1.3648 & -0.9124 & -0.7928 \\
-0.7928 & 0.8965 & -0.7928 & -0.9124 & -1.3648 & -1.3875 & -1.3648 & -0.9124 \\
-0.9124 & -0.7928 & 0.8965 & -0.7928 & -0.9124 & -1.3648 & -1.3875 & -1.3648 \\
-1.3648 & -0.9124 & -0.7928 & 0.8965 & -0.7928 & -0.9124 & -1.3648 & -1.3875 \\
-1.3875 & -1.3648 & -0.9124 & -0.7928 & 0.8965 & -0.7928 & -0.9124 & -1.3648 \\
-1.3648 & -1.3875 & -1.3648 & -0.9124 & -0.7928 & 0.8965 & -0.7928 & -0.9124 \\
-0.9124 & -1.3648 & -1.3875 & -1.3648 & -0.9124 & -0.7928 & 0.8965 & -0.7928 \\
-0.7928 & -0.9124 & -1.3648 & -1.3875 & -1.3648 & -0.9124 & -0.7928 & 0.8965
\end{array}\right]
$$

It is in a circulant form hence if we wish to store the matrix (or other matrices for different values of $n$ ) then we only need to store the first row (or rows). Table 1 gives the first row of $D_{n . k}$ for some selected $n$; with $k=2$. Once the necessary sampled comparisons for a particular matrix size are made by the decision maker, the circulant matrix $D_{n . k}$ is multiplied by the row mean vector $w$ to get the final 
additive $y$ weights. Also, it is necessary to find a way to measure and feedback the consistency of such judgments. The issue is investigated in the next section.

\section{Table 1}

The output of $D_{n, k}$ for different $n$ with $\mathrm{k}=2$

\begin{tabular}{ll}
\hline $\mathrm{n}$ & $\mathrm{K}=2$ \\
\hline 7 & $0.6348,-0.8268,-1.2114,-1.2114,-0.9037,-0.8268$ \\
8 & $0.8965,-0.7928,-0.9124,-1.3648,-1.3648,-0.9124,-0.7928$ \\
9 & $1.1773,-0.7330,-0.8654,-1.4015,-1.5625,-1.5625,-1.4015,-0.8654,-0.7330$ \\
10 & $1.5153,-0.6224,-0.8493,-1.5308,-1.7577,-1.8946,-1.7577,-1.5308,-0.8493,-0.6224$ \\
\hline
\end{tabular}

\subsection{The consistency of Sampled Comparisons}

Consistency is used as a measure of the internal quality of the comparisons made, and as noted by Foster (1994), this quality is a relative concept and has no objective components. However, the study of the consistency of a $k$-diagonal sample provides a useful check on comparisons made and a way to attach some level of confidence to the scores produced by the sample.

\subsubsection{Consistency linear indicator}

Foster (1994) and Al-Dubaibi (1998) proved that the Frobenius distance between a sample matrix and the spaces of consistent sample matrices was denoted by the CLI and given by the following Lemma which follows from Theorem 1.

\section{Lemma 2}

Defining consistency in terms of distances in the linear spaces and having the concept of invariance allow Monte-Carlo simulation methods to be employed.

$$
C L I=\sqrt{\sum\left(a_{i j}^{2}-2 n w^{\prime} y\right)}
$$

By generating large numbers of samples and analyzing the distribution of the CLI, it will be possible to reduce the probability of a decision maker randomly obtaining a specific degree of consistency. Following, we describe our simulation analysis and test the distribution of the CLI for different matrix sizes and different diagonals.

\subsubsection{Results of Computer Simulations}

In determining the distribution of the CLI for $k$-diagonal sampled matrices, computer simulation experiments were carried out using 100,000 randomly-generated diagonal sample decision matrices of size 6 to 50 with different 2-diagonal samples. Random numbers from the set of the category difference scale were generated to form the upper right triangle of the matrices. The rest of the matrices were filled with the appropriate numbers for a pairwise comparisons matrix. We had ensured that there were no level-1 inconsistencies (those in which there is an intransitivity) in the sampled comparisons by generating positive random matrices $a_{i j}>0$ for the appropriate value of $i<j$. Furthermore, we computed the $1 \%$ and $5 \%$ percentile levels for the CLI as this indicates significant consistency. Using this as a measure of consistency shows that it would be improbable, i.e., a chance of one in 100 or a chance of one in 20 for such consistency to occur by chance. It is also shown by Al-Dubaibi (1998) that in generally acceptable consistency occurs when a decision maker's judgments are within this $5 \%$ tail of the distribution of consistency. There are reasonable standards to judge the quality of decision making and hence allows a degree of confidence to be attached to it. We use the Kolmogorov-Smirnov (K-S) goodness-of-fit test using SPSS software system to test for normality as we are dealing with symmetric distributions with small sizes (Yazici \& Yolacan, 2007). The null hypothesis is specified that the sampling distribution is the normal distribution with unspecified mean and variance. The alternative hypothesis is that sampling distribution is not normal. The value of 2-tailed p-value which is the 
observed significance level is obtained to test this hypothesis and has the same meaning as the specified level of significance a. We assume $\mathrm{a}=0.01$ as reported in the literature and hence we accept the null hypothesis is the "2-tailed p-value" is $>0.01$. Also, we find that the null hypothesis is accepted for all $n$. Thus, the sampling distribution of the CLI follows a normal distribution for all $n$ (Alrasheedy, 2003) and shows a selection of the simulation experiments for different $n$ and illustrates the distribution of the CLI. Table 2 shows the 1\% and 5\% significance levels for the CLI for 2-diagonal sampling for $n$ $=6,7, \ldots, 50$.

\subsubsection{Reliability and Feedback}

Once the comparisons have been made, then it is important to feedback internal inconsistencies in the logic to the decision maker. The CLI for the particular matrix is calculated and compared to its corresponding CLI in Table 2 (depending on the number of diagonals) to indicate the quality of the decision making. Another issue is the quality of feedback to the decision maker. The problems are of course inter-linked and will be clear when a computer prototype system is developed.

Table 2

Descriptive statistics and K-S test for 2-diagonal samples

\begin{tabular}{ccccc}
\hline $\mathbf{N}$ & $\mathbf{7}$ & $\mathbf{8}$ & $\mathbf{9}$ & $\mathbf{1 0}$ \\
\hline CLI (5\%) & 10.5357 & 12.4458 & 14.2042 & 15.7228 \\
Mean & 15.9266 & 17.8518 & 19.6847 & 21.3246 \\
St. Dev. & 3.2167 & 3.2439 & 3.2688 & 3.3095 \\
2-tailed P & 0.02804 & 0.4328 & 0.1674 & 0.1817 \\
\hline
\end{tabular}

It is the 5\% significance level that will be implemented in our prototype system later on since we are using 2-diagonal sampling in the software.

\subsection{Numerical example}

In this section, we solve a numerical example and compare our results with those obtained by Ergu et al. (2016) who used a GMIBM technique to analyze the emergency decision making related to the Yushu earthquake. This case dealt with the estimation of the relative assessment of the following eight criteria: casualties, personnel rescued, rescue workers input, materials input, direct economic losses, indirect economic losses, the effectiveness of the physical environment and social benefits. This example was initially solved by Ergu et al. (2016) using the ratio scale from 1/9 to 9. According to Lootsma (1999), there is no difference between the ratio scale and additive scale for the decision maker as far as the input judgment statements are concerned. Then, the reciprocal matrix presented in the cited paper can be transformed into an additive skew-symmetric matrix from -8 to +8 as follows (see Lootsma, 1999):

$$
\begin{aligned}
& \text { Reciprocal matrix (ratio scale) } \\
& {\left[\begin{array}{llllllll}
1 & 5 & 3 & 7 & 6 & 6 & \frac{1}{3} & \frac{1}{4} \\
\frac{1}{5} & 1 & \frac{1}{4} & 5 & 2 & 3 & \frac{1}{2} & \frac{1}{7} \\
\frac{1}{3} & 4 & 1 & 9 & 3 & 6 & 6 & 1 \\
\frac{1}{7} & \frac{1}{5} & \frac{1}{9} & 1 & \frac{1}{2} & \frac{1}{4} & \frac{1}{7} & \frac{1}{8} \\
\frac{1}{6} & \frac{1}{2} & \frac{1}{3} & 2 & 1 & 1 & \frac{1}{5} & \frac{1}{9} \\
\frac{1}{6} & \frac{1}{3} & \frac{1}{6} & 4 & 1 & 1 & \frac{1}{3} & \frac{1}{6} \\
3 & 2 & \frac{1}{6} & 7 & 5 & 3 & 1 & \frac{1}{3} \\
4 & 7 & 1 & 8 & 9 & 6 & 3 & 1
\end{array}\right] \rightarrow}
\end{aligned}
$$

skew-symmetric matrix (additive scale)

$$
\left[\begin{array}{cccccccc}
0 & 4 & 2 & 6 & 5 & 5 & -2 & -3 \\
-4 & 0 & -3 & 4 & 1 & 2 & -1 & -6 \\
-2 & 3 & 0 & 8 & 2 & 5 & 5 & 0 \\
-6 & -4 & -8 & 0 & -1 & -3 & -6 & -7 \\
-5 & -1 & -2 & 1 & 0 & 0 & -4 & -8 \\
-5 & -2 & -5 & 3 & 0 & 0 & -2 & -5 \\
2 & 1 & -5 & 6 & 4 & 2 & 0 & -2 \\
3 & 6 & 0 & 7 & 8 & 5 & 2 & 0
\end{array}\right]
$$


As stated above, the only acceptable solution to the additive pairwise comparisons matrix is the arithmetic mean which is given as $(2.125,-0.875,2.625,-4.375,-2.375,-2,1,3.875)$. Furthermore, taking the row means as exponents of the progression factor $\sqrt{2}$ and normalizing the results to sum to 1 , one can easily obtain the final scores of the alternative options as $(0.178,0.063,0.212,0.019,0.037$, $0.043,0.121,0.327)$. The final scores using the AHP-least square method are $(0.189,0.059,0.205$, $0.018,0.032,0.036,0.153,0.309)$. Now, suppose that the decision maker has been asked to complete 16 comparisons instead of 28 comparisons as in the complete matrix. By using our proposed 2-diagonal sampling scheme, the 2-diagonal sample matrix can be presented as:

$$
A=\left[\begin{array}{cccccccc}
0 & 4 & 2 & * & * & * & -2 & -3 \\
-4 & 0 & -3 & 4 & * & * & * & -6 \\
-2 & 3 & 0 & 8 & 2 & * & * & * \\
* & -4 & -8 & 0 & -1 & -3 & * & * \\
* & * & -2 & 1 & 0 & 0 & -4 & * \\
* & * & * & 3 & 0 & 0 & -2 & -5 \\
2 & * & * & * & 4 & 2 & 0 & -2 \\
3 & 6 & * & * & * & 5 & 2 & 0
\end{array}\right]
$$

The starred entries - treated as zeros by a computer program- are not made, and we wish to complete the comparisons with the assumption that the corresponding completed judgment matrix is as consistent as possible. Then, we are going to find the sample weights by taking the arithmetic row mean $w$. The sample mean is $W=(0.125,-1.125,1.375,-2,-0.625,-0.5,0.75,2)$. Since we have $D_{8,2}$ it is possible to generate the vector $y$ by post-multiplying $D_{8,2}$ by $w$. This gives the additive $y$ weights $(1.760,-1.675,1.079,-4.877,-1.884,-1.037,2.296,4.334)$ which after manipulating we obtain the final scores of criteria as $(0.154,0.047,0.121,0.015,0.043,0.058,0.185,0.375)$. The final result $(Y)$ is close to the row mean of the original one. Furthermore, $B$ is given by $b_{i j}=y_{i}-y_{j}, \forall i, j \notin \Psi$ and can be easily generated. For instance, the value of the comparison between criterion 1 and criterion 4 is $b_{1,4}=y_{1}-y_{4}=1.760-(-4.877)=6.637$. Doing so, the most consistent complete matrix to the sample is given as follows (nearest to two decimals).

$$
\left[\begin{array}{cccccccc}
0 & 4 & 2 & 6.64 & 3.64 & 2.8 & -2 & -3 \\
-4 & 0 & -3 & 4 & 0.21 & -0.64 & -3.97 & -6 \\
-2 & 3 & 0 & 8 & 2 & 2.12 & -1.22 & -3.26 \\
-6.64 & -4 & -8 & 0 & -1 & -3 & -7.17 & -8 \\
-3.64 & -0.21 & -2 & 1 & 0 & 0 & -4 & -6.22 \\
-2.8 & 0.64 & -2.12 & 3 & 0 & 0 & -2 & -5 \\
2 & 3.97 & 1.22 & 7.17 & 4 & 2 & 0 & -2 \\
3 & 6 & 3.26 & 8 & 6.22 & 5 & 2 & 0
\end{array}\right]
$$

It can also be argued that it would be more realistic to obtain integer approximation values to the completed judgment matrix above and to recalculate the weights after considering this rounding. The result of the 2-diagonal sampling technique is successful for this example as can be judged by comparing the weights obtained from those of the original decision matrix. This is supported further by calculating the CLI which indicates good consistency since $C L I=8.385 \leq 12.446$. Furthermore, the number of pairwise comparisons is reduced relative to Ergun et al. (2016). 


\section{Conclusions}

The pairwise comparisons technique has been long used in decision making, but it has a major drawback when evaluating a large number of alternatives. The current paper proposed a sampling technique which is based on optimizing consistency with respect to any permutation of invariant inner product on the linear space of skew-symmetric matrices. It exploited special properties of the diagonal samples and calculations of circulant matrices to provide simple formulae for finding scores and consistencies. Using the diagonal sampling technique, usually with $k=2$, together with explicit calculations for the matrices $D_{n . k}=-\frac{1}{n} S R Q_{1}^{-1}$ gives a fast and effective way to complete the comparisons. We have demonstrated that the solution is in the form of a circulant matrix which provides a compact means of storage since we only need to store the first row. Furthermore, we have developed look-up tables for the CLI for 2-diagonal samples for decision alternatives between 6 and 50. The paper also addressed the issues of reliability and employed simulations to develop consistency standards for comparisons. The fundamental importance of this matter is that it gives reasonable standards to judge the quality of decision making and hence allows a degree of confidence to be attached to the consistency of a sample. We have carefully considered the accuracy of the calculations since the data calculated are directly utilized if there is a decision support system to be built. In essence, the approaches of this article represent a new sampling method which is fundamentally different from other attempts (Carmone et al., 1997; Ra, 1999) and dramatically reduces the number of comparisons to be made from $n(n-1) / 2$ to $2 n$ while maintaining the same quality of results. To show the effectiveness of the method and the overall reduction in comparisons between the traditional technique and our proposed method an example of the literature is solved (Ergu et al., 2016).

\section{References}

Akarte, M. M., Surendra, N. V., Ravi, B., \& Rangaraj, N. (2001). Web based casting supplier evaluation using analytical hierarchy process. Journal of the Operational Research Society, 52(5), 511-522.

Al-Dubaibi, A. (1998). The Best Model for Pairwise Comparisons. Unpublished Ph.D. Thesis, Brunel University, UK.

Alrasheedy, M. (2003). Solving multi-criteria decision problems using pairwise comparisons with a web-based prototype decision system. Unpublished Ph.D. Thesis, Brunel University, UK.

Al Salem, A. (2017). Managing consistency and consensus in group decision making with incomplete fuzzy preference relations. Montreal, Quebec, Canada.

Barzilai, J. (1997). Deriving weights from pairwise comparison matrices. Journal of the operational research society, 48(12), 1226-1232.

Barzilai, J., Cook, W. D., \& Golany, B. (1987). Consistent weights for judgements matrices of the relative importance of alternatives. Operations research letters, 6(3), 131-134.

Benítez, J., Delgado-Galván, X., Izquierdo, J., \& Pérez-García, R. (2015). Consistent completion of incomplete judgments in decision making using AHP. Journal of Computational and Applied Mathematics, 290, 412-422.

Bochkov, A., \& Zhigirev, N. ( 2014). The analytic hierarchy process modification for decision making under uncertainty. Reliability: Theory and Applications, 9(2), 36-45.

Bozoki, S. (2010). Incomplete pairwise compárison matrices and connectedness. The Corvinus University of Budapest, Department of Operations Research and Decision Systems.

Bozoki, S., Fulop, J., \& Ronyai, L. (2010). On optimal completion of incomplete pairwise comparison matrices. Mathematical and Computer Modelling, 52(1), 318-333.

Carmone, F., Kara, S., \& Zanakis, S. (1997). A Monte Carlo investigation of incomplete pairwise comparison matrices in AHP. European Journal of Operational Research, 102(3), 538-553. 
Chen, K., Kou, G., Tarn, J., \& Song, Y. (2015). Bridging the gap between missing and inconsistent values in eliciting preference from pairwise comparison matrices. Annals of Operations Research, 235(1), 155-175.

Davis, P. J. (1994). Circulant matrices. AMS Chelsea Publishing.

Ergu, D., Kou, G., Peng, Y., \& Zhang, M. (2016). Estimating the missing values for the incomplete decision matrix and consistency optimization in emergency management. Applied Mathematics Modelling, 40(1), 254-267.

Fedrizzi, M., \& Giove, S. (2007). Incomplete pairwise comparison and consistency optimization. European Journal of Operational Research, 183(1), 303-313.

Foster, W. (1994). Linear models for pairwise comparisons and measures of agreement. Unpublished Ph.D. Thesis, Brunel University, UK.

Foster, W. (2001). Reliable Decisions Using Pairwise Comparisons. $\sigma^{\text {th }}$ Conference of the ISDSS. Uxbridge, UK.

Foster, W. (1997). Sampling Pairwise Comparisons. Unpublished Technical Report. Brunel University, UK.

Han, S. (2016). How can we handle too many criteria/alternatives?: A study on AHP structural design. NUCB Journal of Economics and Information Science, 60(2), 103-112.

Harker, P. (1987a). Incomplete pairwise comparisons in the analytic hierarchy process. Mathematical Modelling, 9(11), 837-848.

Harker, P. T. (1987 b). Alternative modes of questioning in the analytic hierarchy process. Mathematical Modelling, 9(3-5), 353-360.

Harker, P. (1987c). Shortening the comparison process. Mathematical Modelling, 8, 139-141.

Hotman, E. (2005). Base reference analytical hierarchy process for engineering process selection. International Conference on Knowledge-Base and Intelligent Information and Engineering Systems, (págs. 184-190). Springer, Berlin, Heidelberg.

Inti, S., \& Tandon, V. (2017). Application of fuzzy preference-Analytic Hierarchy Process logic in evaluating sustainability of transportation infrastructure requiring multi-criteria decision making. Journal of Infrastructure Systems, 23(4). https://doi.org/10.1061/(ASCE)IS.1943-555X.0000373

Jalao, E., Wu, T., \& Shunk, D. (2014). An intelligent decomposition of pairwise comparison matrices for large-scale decisions. European Journal of Operational Research, 238(1), 270-280.

Joneghani, S.Z., \& Joneghani, H.Z.(2018). Identification and ranking the effective factors for export promotion in pharmaceutical companies , Management Science Letters, 9(3),357-364.

Kahraman, C., Suder, A. \& Bekar, E.T.(2016). Fuzzy multi-attribute consumer choice among health insurance options, Technological and Economic Development of Economy, 22(1),1-20.

Koczkodaj, W., \& Szybowsky. (2015). Pairwise comparisons simplified. Journal of Applied Mathematics and Computation, 253, 387-394.

Kou, G., Ergu, D., Lin, C., \& Chen, Y. (2016). The pairwise matrix in multiple criteria decision making. Technological and economic development of an economy, 22(5), 738-765.

Lolli, F., Ishizaka, A., Gamberini, R. (2014). New AHP-based approaches for multi-criteria inventory classification, International Journal of Production Economics, 156, 62-74.

Lootsma, J. (1999). Multi-Criteria Decision Analysis via Ratio and Difference Judgment. The Netherlands: Kluwer Academic Publishers, The Netherlands, 50-140

Makui, A., Fathi, M., Narenji, M. (2010). Interval weighted comparison matrices- A review. International Journal of Industrial Engineering and Production Research, 20(4), 139-156.

Peng, Y., Kou, G., Ergu, D., \& Shi, Y. (15-18 de June de 2011). Handling missing survey data in AHP. XI Symposium ISAHP 2011.Sorrento, Naples, Italy.

Ra, J. (1999). Chainwise Paired Comparisons. Decision Sciences, 30(2), 581-598.

Saaty, T. (1980). The analytical hierarchy process. New York: John Wiley. 
Ramik, J. (2013). Incomplete pairwise comparison matrix and its application to the ranking of alternatives. Multiple Criteria Decision Making Journal, 8, 114-128.

Schmidt, K., Aumann, I., Hollander, I., Damm, K., \& von der Schulenburg, J. M. G. (2015). Applying the Analytic Hierarchy Process in healthcare research: A systematic literature review and evaluation of reporting. BMC medical informatics and decision making, 15(1), 112.

Song, B., \& Kang, S. (2016). A method of assigning weights using a ranking and nonhierarchy comparison. Advances in Decision Sciences, Vol. 2016, Article ID 8963214, 9 pages.

Srdjevic, B., Srdjevic Z, \& Blagojevic, B. (2014). First-level transitivity rule method for filling in incomplete pair-wise comparison matrices in the analytic hierarchy process. Applied Mathematics and Information Sciences, 8(2), 459-467.

Tekile, H., \& Bozoki, S. (2017). Incomplete pairwise comparison matrices in multicriteria decision making and ranking. Master of Science Thesis, Central European University, Department of Mathematics and its Applications, Budapest, Hungary.

Toloie-Eshlaghy, A., \& Homayonfar, M. (2011). MCDM methodologies and applications: a literature review from 1999 to 2009. Research Journal of International Studies, 21, 86-137.

Triantaphyllou, E. (1999). Reduction of pairwise comparisons in decision making via a duality approach. Journal of Multi-Criteria Decision Analysis, 8(6), 299-310.

Um, W. (2016). Supplier evaluation and selection using method and uncertainty theory. Proceedings of the Second World Congress on Mechanical, Chemical, and Material Engineering. Budapest, Hungary.

Wang, J., Lan, J., Ren, P., \& Luo, Y. (2012). Some programming models to derive priority weights from additive interval fuzzy preference relation. Knowledge-Based Systems, 27, 69-77.

Wang, Z. J. \& Li, K. (2015). A multi-step goal programming approach for group decision making with incomplete interval additive reciprocal comparison matrices. European Journal of Operational Research, 242(3), 890-900.

Wedley, W. (1993). Consistency prediction for incomplete AHP matrices. Mathematical and Computer Modelling, 17(4-5), 151-161.

Wedley, W., Schoner, B., \& Tang, T. (1993). Starting rules for incomplete comparisons in the analytic hierarchy process. Math. Comput. Modeling, 17(4/5), 93-100.

Xu, Y., Patnayakuni, R., \& Wang, H. (2013). Logarithmic least squares method to priority for group decision making with incomplete fuzzy preference relations. Applied Mathematical Modelling, 37 (4), 2139-2152.

Yazici, B., Yolacan, S. (2007). A comparison of various tests of normality. Journal of Statistical Computation and Simulation, 77(2), 175-183.

Zhang, G., Dong, Y., \& Xu, Y. (2012). Linear optimization modeling of consistency issues in group decision making based on fuzzy preference relations, Expert Systems with Applications, 39(3), 2415-2420.

Zhou, X., Hu, Y., Deng, Y., Chan, F., \& Ishizaka, A. (2018). A Dematel- based completion method for incomplete pairwise comparison matrix in AHP. Annals of Operations Research, 271(2), 10441066.

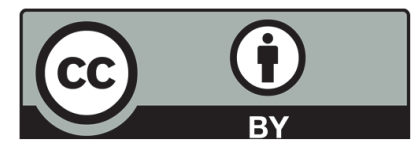

(C) 2019 by the authors; licensee Growing Science, Canada. This is an open access article distributed under the terms and conditions of the Creative Commons Attribution (CC-BY) license (http://creativecommons.org/licenses/by/4.0/). 